\title{
ACK1 promotes hepatocellular carcinoma progression via downregulating WWOX and activating AKT signaling
}

\author{
BINHUI XIE $^{1 *}$, QINSHAN ZEN $^{1 *}$, XIAONONG WANG $^{1}$, XIAO HE $^{1}$, \\ YUANKANG XIE ${ }^{1}$, ZIXIANG ZHANG ${ }^{2}$ and HEPING LI $^{3}$ \\ Departments of ${ }^{1}$ Hepatobiliary Surgery, ${ }^{2}$ Gastroenterology, First Affiliated Hospital \\ of Gannan Medical University, Ganzhou 341000; ${ }^{3}$ Department of Interventional Radiology, \\ the First Affiliated Hospital of Sun Yat-sen University, Guangzhou 510080, P.R. China \\ Received December 29, 2014; Accepted February 19, 2015
}

DOI: $10.3892 /$ ijo.2015.2910

\begin{abstract}
Several studies have revealed that ACK1 is upregulated in various cancers and promotes tumor progression. However, the role and mechanism of ACK1 in hepatocellular carcinoma (HCC) remains unknown. In this study, the expression of ACK1 was assessed in several cell lines and 150 pairs of HCC and adjacent noncancerous liver tissues. The protein expression of p-ACK1 and WWOX were detected by immunohistochemistry to evaluate their correlation with ACK1. Flow cytometry, caspase 3/7 activity assay, BrdU cell proliferation assay, MTT assay and Transwell assay were used to detect apoptosis, proliferation, invasion and migration of HCC cells. The regulatory effect of ACK1 on WWOX, AKT, p-AKT, MMP2 and MMP9 in HCC cells was confirmed by immunoblotting. We found that ACK1 was more highly expressed in $\mathrm{HCC}$ tissues than in non-HCC tissues, and overexpression of ACK1 was correlated with clinicopathological features of poor prognosis. Clinical analysis demonstrated that ACK1 is an independent prognostic marker for predicting overall survival and disease-free survival of HCC patients. Pearson's correlation coefficient analysis indicated that ACK1 was positively associated with p-ACK1 and was negatively associated with WWOX expression. In vitro studies showed that knockdown of ACK1 promoted HCC cell apoptosis and repressed HCC cells invasion, migration and proliferation. Furthermore, knockdown of ACK1 resulted in upregulation of WWOX and
\end{abstract}

Correspondence to: Dr Zixiang Zhang, Department of Gastroenterology, First Affiliated Hospital of Gannan Medical University, Ganzhou 341000, P.R. China

E-mail: zhangzx1964@163.com

Dr Heping Li, Department of Interventional Radiology, the First Affiliated Hospital of Sun Yat-sen University, Guangzhou 510080, P.R. China

E-mail: 1ihp1979@163.com

${ }^{*}$ Contributed equally

Key words: ACK1, p-ACK1, hepatocellular carcinoma, WWOX, AKT inactivation of AKT signaling. In this study, we also found that knockdown of ACK1 resulted in the downregulation of MMP2 and MMP9 in HCC. Our results indicate that ACK1 is an independent prognostic marker and promotes HCC progression via downregulating WWOX and activating AKT signaling.

\section{Introduction}

Hepatocellular carcinoma (HCC) is one of the most frequent malignant tumors worldwide and ranks as the second most common cause of cancer-related death in China (1-3). In recent years, in line with the rising morbidity of hepatitis $\mathrm{C}$ virus infection, the incidence of HCC is increasing in many western countries including the United States (4-6). At present, due to the high recurrence rate and early metastasis, the prognosis of the HCC patients remains very poor $(7,8)$. Hence, understanding the pathogenesis of HCC and exploring prognostic markers and therapeutic targets is imperative for the treatment of HCC.

Activated $\mathrm{Cdc} 42$ associated kinase (ACK1, also known as TNK2) was originally considered as a Cdc42-interacting protein, and was suggested to be a Cdc42 effector $(9,10)$. In addition to interaction with Cdc42, other ACK1 interacting partners include clathrin, ubiquitin, Grb2 and Nedd4-2 E3 ligase $(11,12)$. As a ubiquitously expressed non-receptor tyrosine kinase, ACK1 has emerged as an important transducer of variety of extracellular signals (13). Importantly, ACK1 gene amplification can cause ACK1 phosphorylation (p-ACK1) and auto-activation, which results in the activation of ACK1 signal transduction $(14,15)$. Activated ACK1 senses extracellular signals through interacting with activated receptor-tyrosine kinases including AKT, EGFR, HER2 and MERTK $(15,16)$. Such interactions result in ACK1-activated signal transduction through the activation of multiple downstream effectors including androgen receptor (AR) (17). Through these ACK1 activated signaling networks, ACK1 participates in cell survival, invasion, migration and tumorigenesis (18).

WW domain-containing oxidoreductase (WWOX) is a newly found tumor suppressor protein (19). Extensive research indicates that WWOX is downregulated in various tumor types including HCC (19-22). Notably, WWOX is a target of ACK1 signaling in prostate cancer and ACK1 could negatively 
regulate WWOX protein expression and promote WWOX degradation (23).

The AKT signaling pathway, is critical in promoting cell proliferation, invasion and migration, and is frequently dysregulated in multiply tumor types (24). Furthermore, it has been reported that AKT signaling plays a key role in facilitating MMP2 and MMP9 expression, which are important factors in the progression of HCC (25). Noteworthy, while AKT signaling pathway is understood to a broad extent in several cancers, new study evidence has emerged demonstrating that AKT signaling activation can occur in PI3K-independent pattern (26). Several studies have already identified a novel mechanism of ACK1 mediated AKT activation, which leads to AKT signaling translocation to the cell plasma membrane and subsequent molecule activation $(23,26)$. However, the mechanism of ACK1-mediated AKT activation in HCC is not clear.

Several studies have implicated ACK1 amplification and high-expression in carcinogenesis of multiple tissue types such as lung, breast and prostate (27-29). Overexpression level of ACK 1 correlates with tumor invasion and migration in renal and pancreatic cancers $(30,31)$. Importantly, ACK1 activationinduced robust AKT signaling activation has been found in various tumor types (23). However, the role and mechanisms involved in ACK1 are still unknown in human HCC.

In this study, we demonstrate that ACK1 is an independent prognostic marker for predicting both the overall and diseasefree survival of patients with HCC. ACK1 can negatively regulate WWOX expression in HCC cells. Knockdown of ACK1 resulted in upregulation of WWOX and inactivation of AKT signaling. In this study, we also found that knockdown of ACK1 resulted in the downregulation of MMP2 and MMP9 in HCC. Notably, our results indicate that ACK1 is a candidate oncogene in $\mathrm{HCC}$ because it plays an important role in hepatocarcinogenesis.

\section{Materials and methods}

Cell culture and clinical samples. The immortalized human liver cell line L02 was obtained from the American Type Culture Collection (Manassas, VA, USA). HCC cell lines SMMC-7721, Huh-7, HepG2, BEL-7402 and MHCC-97H were purchased from the Type Culture Collection of the Chinese Academy of Sciences (Shanghai, China). All the cell lines were maintained in Dulbecco's modified Eagle's medium (Gibco, USA) containing 10\% fetal bovine serum (Gibco, USA) and cultured in a humidified incubator containing $5 \%$ $\mathrm{CO}_{2}$ at $37^{\circ} \mathrm{C}$.

A total of 150 pairs of HCC and corresponding adjacent non-tumorous liver samples $(>2.0 \mathrm{~cm}$ from the resection margin) were obtained from the patients who underwent hepatectomy between January 2005 and June 2009 at First Affiliated Hospital of Gannan Medical University. The tumor stages were classified according to the new 7th edition (TNM-7) of the American Joint Committee on Cancer (AJCC)/ International Union Against Cancer (UICC) TNM system (32). The demographic features and clinicopathologic data of these patients are shown in Table I. Our study enrolled 118 males and 32 females with a median age of 52 years. All samples were collected immediately after hepatectomy, snap-frozen in liquid nitrogen, and stored at $-80^{\circ} \mathrm{C}$ until use. All recruited patients provided written informed consent before surgical resection, and all the protocols were approved by the Ethics Committee of First Affiliated Hospital of Gannan Medical University according to the 1975 Declaration of Helsinki.

Quantitative reverse transcription-polymerase chain reaction (qRT-PCR). Total RNA was isolated from cell lines using TRIzol reagent (Invitrogen, USA). The RNA samples were reverse transcribed into cDNA with a RevertAid Premium First Strand cDNA Synthesis kit (Fermentas, Canada). qRT-PCR was done in an ABI 7500 system using the SYBR ${ }^{\circledR}$ Premix Ex $\mathrm{Taq}^{\mathrm{TM}}$ II (Takara, Japan). The following primers were used: ACK1 sense primer: 5'-AGAGCCTGAAGACACGCACC-3'; antisense primer: 5'-GGATCTGACTGCCGTTGAGG-3'. $\beta$-actin sense primer: 5'-GGGAAATCGTGCGTGACAT-3'; antisense primer: 5'-CTGGAAGGTGGACAGCGAG-3'. Three experimental replicates were performed.

Western immunoblotting. The primary rabbit anti-ACK1 polyclonal antibody (ab135672), primary rabbit anti-p-ACK1 polyclonal antibody (ab74091) and primary rabbit anti-WWOX polyclonal antibody (ab189410) were purchased from Abcam (Cambridge, UK). The primary rabbit anti-Ki-67 polyclonal antibody (sc-15402), rabbit anti-AKT polyclonal antibody (sc-8312, which can be used to detect total proteins of AKT1, AKT2 and AKT3), rabbit anti-p-AKT polyclonal antibody (sc-293095, which can be used to detect phosphorylated AKT1, AKT2 and AKT3), rabbit anti-MMP2 polyclonal antibody (sc-10736), rabbit anti-MMP9 polyclonal antibody (sc-10737) and rabbit anti- $\beta$-actin polyclonal antibody (sc-130656) were purchased from Santa Cruz Biotechnology (Santa Cruz, CA, USA). The secondary goat anti-rabbit antibody (sc-2004) was also obtained from Santa Cruz Biotechnology. The blots were examined with the secondary antibody conjugated with HRP and reactions were visualized using the HyGLO HRP detection kit from Diagenode Inc. (Denville, NJ, USA).

Immunohistochemical staining. Immunohistochemical staining was performed on paraformaldehyde-fixed paraffin sections. The ACK1, WWOX and p-ACK1 primary antibodies were used in the immunohistochemistry assays. Immunohistochemical staining was performed as previous reported (25). Immunostaining intensity was evaluated as four grades: 0 , negative; 1 , weak; 2 , moderate; 3 , strong. The percentage of positive cells was categorized as grades: $0,0 \%$; $1,1-10 \% ; 2,11-50 \% ; 3,51-80 \%$; and $4,>80 \%$. The immunostaining intensity and average percentage of positive cells were evaluated for ten independent high magnification fields. By multiplying the staining intensity and the percentage of positive cells, the final weighed expression score was obtained $(0-12)$.

RNAi transfection. The ACK1 shRNA and scrambled shRNA vector $\mathrm{pRS}$ were purchased from OriGene Technologies Inc. (Rockville, MD, USA). ACK1 unique 29mer shRNA constructs in pRS Vector was transfected into MHCC-97H cells using TurboFectin Transfection Reagent purchased from OriGene Technologies Inc. as ACK1-shRNA MHCC-97H cells. The non-effective 29-mer scrambled shRNA cassette in pRS Vector was transfected into MHCC-97H cells as the control cells. 
Cell proliferation and viability assays. For the HCC cell proliferation assay, tumor cells were seeded into 96-well plates at $5 \times 10^{3}$ cells per well for $24 \mathrm{~h}$ and performed using a Cell Proliferation ELISA, BrdU (5-bromodeoxyuridine) (chemiluminescent) (Roche, Indianapolis, IN, USA). The cell viability of HCC cells was determined using 3-(4, 5-dimethylthiazol2-yl)-2, 5-diphenyltetrazolium bromide (MTT) assay. HCC cells were seeded in a 96-well plate. The absorbance of the samples was measured at 24, 48 and $72 \mathrm{~h}$.

Cell apoptotic assays. An Annexin V-FLUOS Staining kit (Roche) was used to determine the level of cell apoptosis, according to the manufacturer's protocols. The caspase 3/7 activity assay was performed using an Apo-ONE ${ }^{\circledR}$ Homogeneous Caspase-3/7 Assay (Promega, WI, USA), as described in a previous study (24).

Statistical analysis. Statistical analysis was performed using the SPSS 16.0 statistical software package (SPSS Inc., Chicago, IL, USA). A two-tailed Student's t-test, a Kaplan-Meier plot, a log-rank test, a Spearman correlation coefficient analysis, a Chi-square test or Fisher's exact test was used to evaluate statistical significance. Independent prognostic factors were assessed by the Cox proportional hazards stepwise regression model. Data are shown as the mean \pm SEM. P-values were two-sided, and a P-value of $<0.05$ was considered to indicate statistical significance.

\section{Results}

Expression level of ACK1 in cell lines. We first detected the expression level of ACK1 in L02, SMMC-7721, Huh-7, HepG2, BEL-7402 and MHCC-97H cell lines using qRT-PCR and immunoblotting. We found that the expression level of ACK1 mRNA in immortalized nontumorigenic human hepatocyte cell line L02 was significantly lower than that in HCC cell lines including SMMC-7721, Huh-7, HepG2, BEL-7402 and MHCC-97H ( $\mathrm{P}<0.01$, respectively, Fig. 1A). Furthermore, MHCC-97H expressed the highest expression level of ACK1 mRNA in HCC cell lines among the five HCC cell lines (Fig. 1A). Our results of immunoblotting assay also verified these findings (Fig. 1B). Thereby, MHCC-97H cells were used in ACK1 knockdown experiment.

Clinical significance of ACK1 expression in HCC samples. To explore the clinical significance of ACK1 in HCC, we examined the expression level of ACK1 in 150 pairs of HCC and matched tumor-adjacent liver tissues using qRT-PCR, immunoblotting and immunohistochemical staining, and found that the expression level of ACK1 was significantly higher in the HCC tissues than that in the noncancerous tissues $(\mathrm{P}<0.01$, respectively, Fig. 2A-C). To further investigate the clinical role of ACK1 in $\mathrm{HCC}$, we determined the correlations of the ACK1 protein expression with clinicopathological characteristics, including patient gender, age, HBsAg, AFP level, tumor size, cirrhosis, capsule formation, Edmondson-Steiner grade, tumor number, vascular invasion and TNM stage. The median expression level of ACK1 protein was used as the cutoff point to divide into low-expressing and high-expressing groups. In this study, the expression level of ACK1 was evidently correlated with the
Table I. Correlations between ACK1 expression and clinicopathologic features in HCC.

\begin{tabular}{|c|c|c|c|c|}
\hline \multirow[b]{2}{*}{ Characteristics } & \multirow[b]{2}{*}{$\mathrm{n}$} & \multicolumn{2}{|c|}{ ACK1 protein } & \multirow[b]{2}{*}{ P-value } \\
\hline & & High & Low & \\
\hline \multicolumn{5}{|l|}{ Gender } \\
\hline Female & 32 & 20 & 12 & 0.162 \\
\hline Male & 118 & 55 & 63 & \\
\hline \multicolumn{5}{|l|}{ Age (year) } \\
\hline$\leq 45$ & 42 & 17 & 25 & 0.203 \\
\hline$>45$ & 108 & 58 & 50 & \\
\hline \multicolumn{5}{|l|}{ HBsAg status $^{\mathrm{a}}$} \\
\hline Negative & 32 & 13 & 19 & 0.319 \\
\hline Positive & 118 & 62 & 56 & \\
\hline \multicolumn{5}{|l|}{ Cirrhosis } \\
\hline No & 48 & 22 & 26 & 0.600 \\
\hline Yes & 102 & 53 & 49 & \\
\hline \multicolumn{5}{|l|}{$\operatorname{AFP}(\mu \mathrm{g} / \mathrm{l})^{\mathrm{b}}$} \\
\hline$\leq 400$ & 67 & 29 & 38 & 0.189 \\
\hline$>400$ & 83 & 46 & 37 & \\
\hline \multicolumn{5}{|l|}{ Tumor size } \\
\hline$\leq 5 \mathrm{~cm}$ & 55 & 26 & 29 & 0.735 \\
\hline$>5 \mathrm{~cm}$ & 95 & 49 & 46 & \\
\hline \multicolumn{5}{|l|}{ Tumor number } \\
\hline Single & 118 & 51 & 67 & $0.002^{\mathrm{c}}$ \\
\hline Multiple & 32 & 24 & 8 & \\
\hline \multicolumn{5}{|l|}{ Tumor capsule } \\
\hline Complete & 35 & 13 & 22 & 0.122 \\
\hline Incomplete & 115 & 62 & 53 & \\
\hline \multicolumn{5}{|c|}{ Vascular invasion } \\
\hline No & 128 & 57 & 71 & $0.002^{\mathrm{c}}$ \\
\hline Yes & 22 & 18 & 4 & \\
\hline \multicolumn{5}{|c|}{ Edmondson grade } \\
\hline $\mathrm{I} / \mathrm{II}$ & 91 & 37 & 54 & $0.007^{\mathrm{c}}$ \\
\hline III/IV & 59 & 38 & 21 & \\
\hline \multicolumn{5}{|l|}{ TNM stage } \\
\hline $\mathrm{I} / \mathrm{II}$ & 96 & 38 & 58 & $0.001^{\mathrm{c}}$ \\
\hline III/IV & 54 & 37 & 17 & \\
\hline
\end{tabular}

${ }^{\mathrm{a}} \mathrm{HbsAg}$, hepatitis B surface antigen; ${ }^{\mathrm{b}} \mathrm{AFP}, \alpha$-fetoprotein; ${ }^{\mathrm{c}}<0.05$.

Edmondson-Steiner grade, tumor number, vascular invasion and TNM stage $(\mathrm{P}<0.05$, respectively). However, no significant correlation was found between the expression of TPX2 and patients gender, age, HBsAg, AFP level, tumor size, cirrhosis and capsule formation ( $\mathrm{P}>0.05$, respectively). These results are listed in Table I.

ACK1 expression is an independent prognostic factor for $\mathrm{HCC}$. In our study, the median expression level of ACK1 protein 


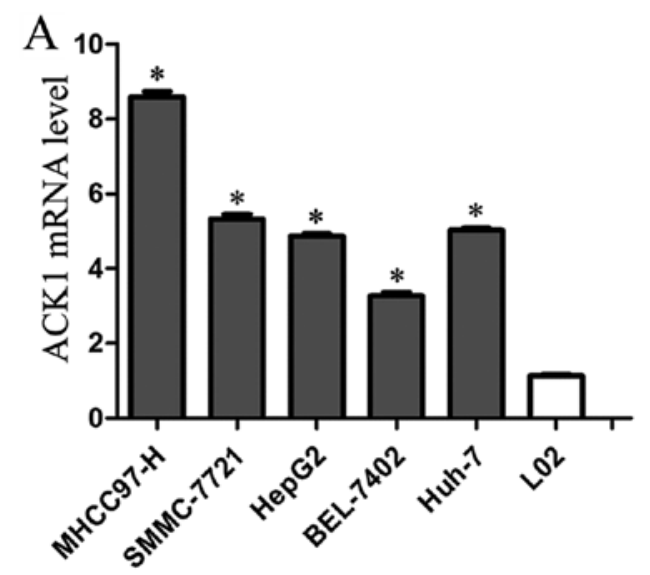

B

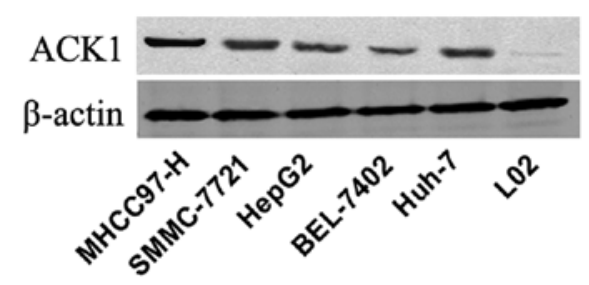

Figure 1. The expression of ACK1 in cell lines. (A) ACK1 mRNA expression in the immortalized normal human liver cell line L-02 and five hepatoma cell lines (SMMC-7721, BEL-7402, Huh-7, HepG2 and MHCC-97H) using qRT-PCR ( $\mathrm{n}=3$, "P<0.01, vs. L-02 group, respectively). (B) ACK1 protein expression in the immortalized normal human liver cell line L-02 and five hepatoma cell lines (SMMC-7721, BEL-7402, Huh-7, HepG2 and MHCC-97H) using immunoblotting $(\mathrm{n}=3, \mathrm{P}<0.01)$.

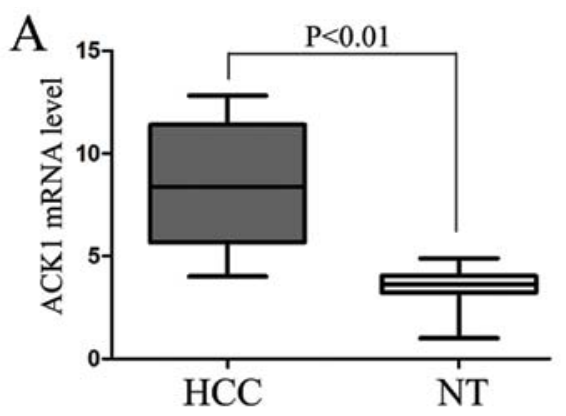

B

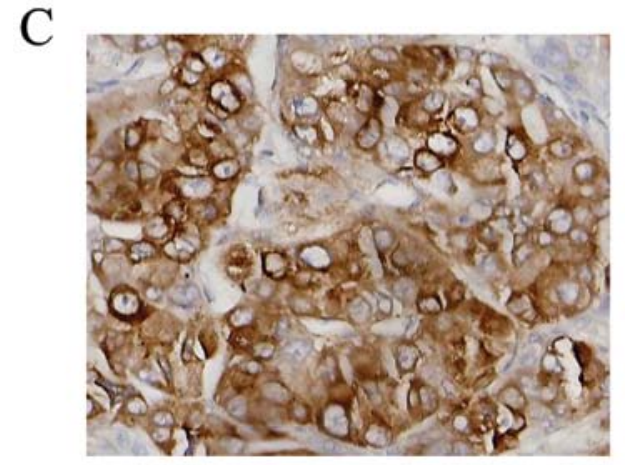

HCC tissue

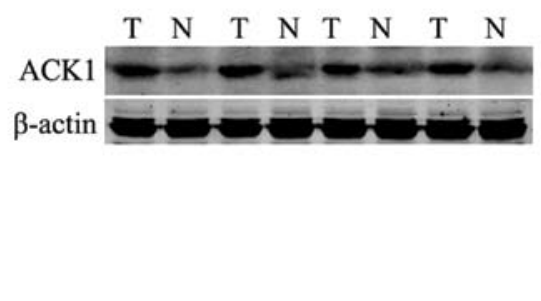

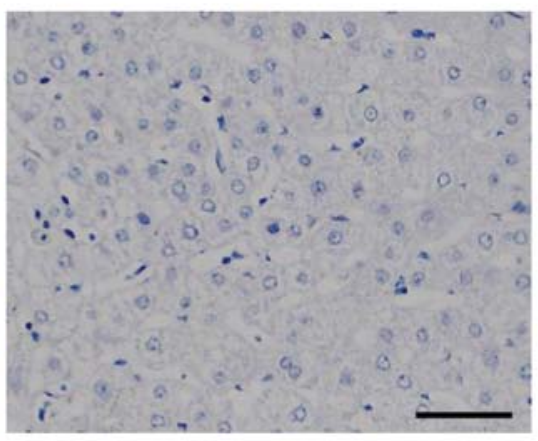

Tumor-adjacent tissue

Figure 2. The expression of ACK1 in HCC tissues. (A) ACK1 mRNA expression in hepatocellular carcinoma tissues (HCC) and corresponding adjacent nontumorous liver tissues (NT) using qRT-PCR $(\mathrm{n}=150, \mathrm{P}<0.01)$. (B) ACK1 protein expression in hepatocellular carcinoma tissues $(\mathrm{T})$ and corresponding adjacent non-tumorous liver tissues $(\mathrm{N})$ using immunoblotting $(\mathrm{n}=150, \mathrm{P}<0.01)$. (C) ACK1 protein expression in hepatocellular carcinoma tissues and corresponding adjacent no tumorous liver tissues using immunohistochemical staining $(\mathrm{n}=150, \mathrm{P}<0.01)$ (scale bar, $50 \mu \mathrm{m})$.

was used as the cutoff point to divide into low-expressing and high-expressing groups for HCC patients' survival. The HCC patients with high ACK1 expression had evidently reduced overall survival and disease-free survival. The 5 -year overall survival rate of the low ACK1 expressing group was 69.0\%, which was evidently higher than that of the high-expressing group (36.9\%, $\mathrm{P}<0.01)$ (Fig. 3A). The 5-year disease-free survival rate of the low ACK1 expressing group was 55.6\%, which was significantly higher than that of the high-expressing group $(20.0 \%, \mathrm{P}<0.01)$ (Fig. 3B). The univariate analysis showed that hepatitis B surface antigen (HBsAg) status, tumor number, vascular invasion, Edmonson-Steiner classification, TNM stage and ACK1 expression were the prognosis factors for HCC patients (Table II). In a multivariate analysis model, the expression level of ACK1 was evidently associated with overall survival (HR 2.523; 95\% CI, 1.496-4.255; $\mathrm{P}<0.01$ ) and disease-free survival (HR 2.318; 95\% CI, 1.479-3.634; $\mathrm{P}<0.01$ ) (Table III). Our results indicated that the ACK1 expression level was an independent prognosis factor in HCC patients.

ACK1 is positively associated with $p-A C K 1$ and negatively associated with WWOX expression in HCC samples. To 

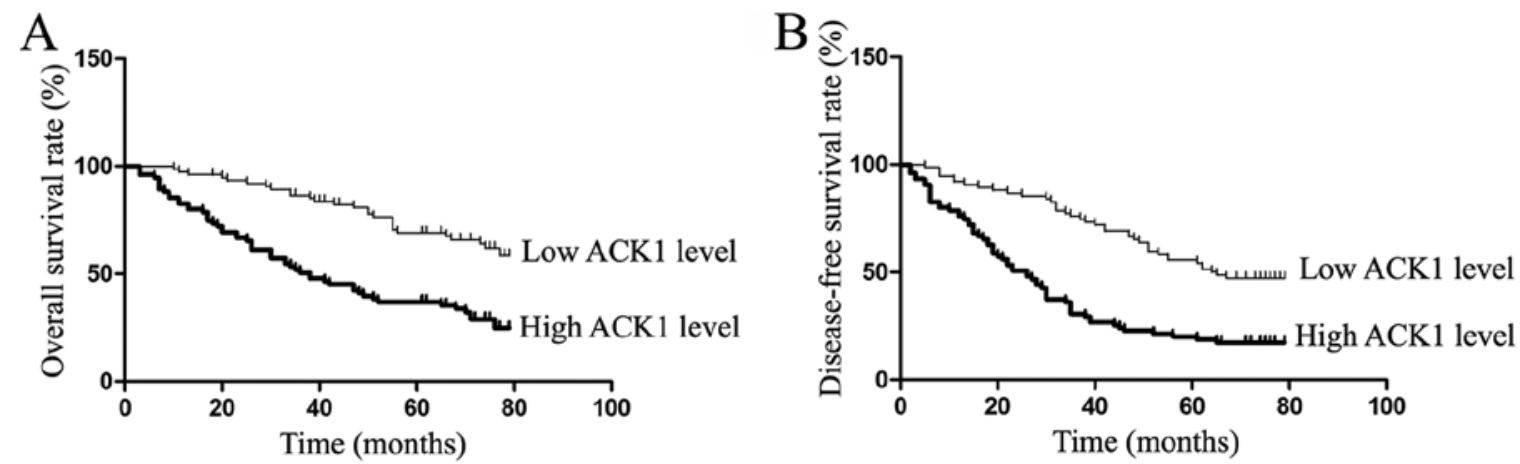

Figure 3. Kaplan-Meier survival curves according to ACK1 protein expression level in HCC patients. (A) Overall survival for ACK1 protein expression (log-rank, $\mathrm{P}<0.01)$. (B) Disease-free survival for ACK1 protein expression (log-rank, $\mathrm{P}<0.01)$.
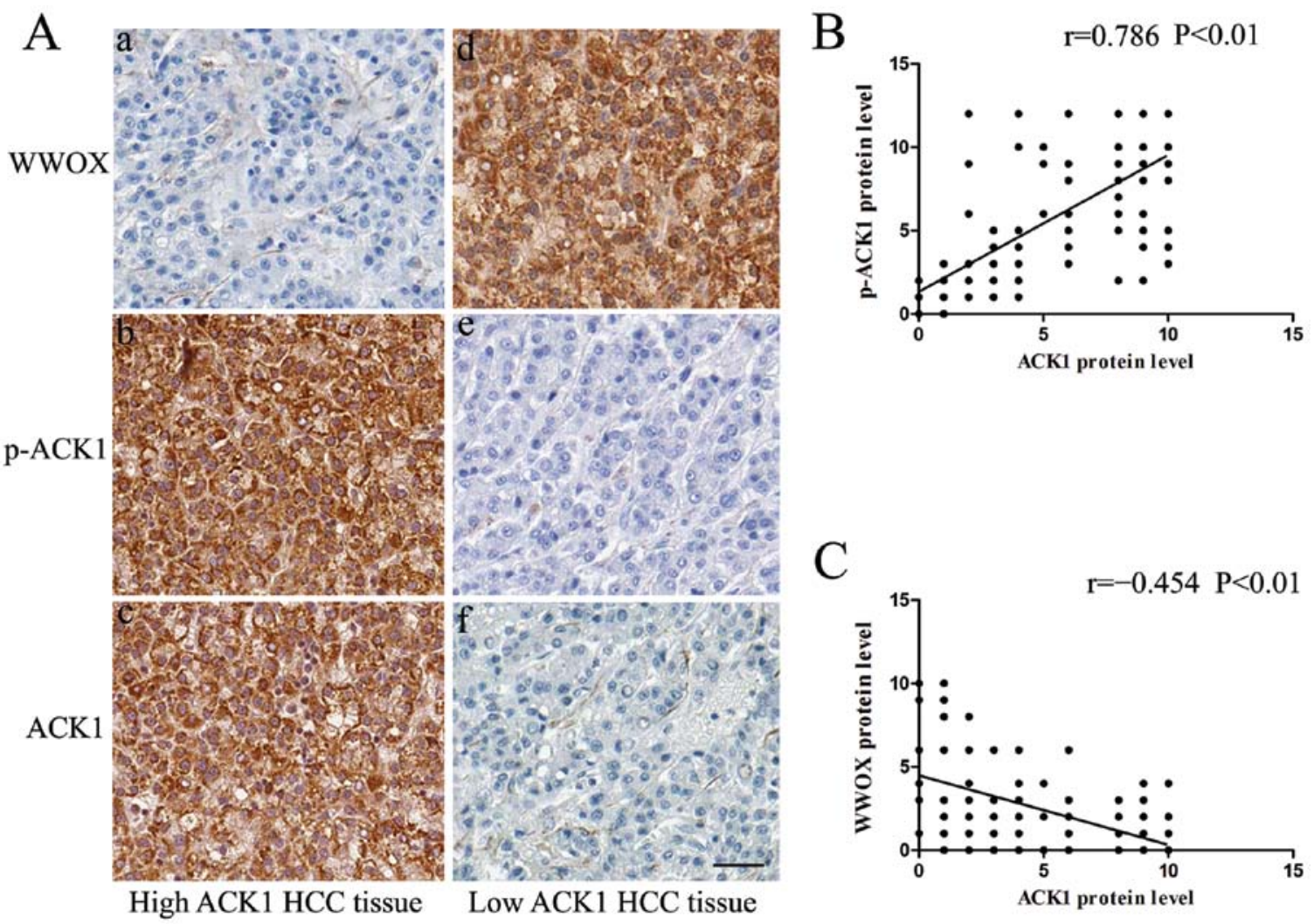

Figure 4. Immunohistochemical staining of ACK1, p-ACK1 and WWOX in hepatocellular carcinoma tissues (HCC). (A) In cases of high ACK1 protein expression (c), there was strong p-ACK1 protein expression (b), whereas there was no detectable WWOX protein expression (a) in the same tissue section. Similarly, in the case of low ACK1 protein expression (f), there was no detectable p-ACK1 protein expression, whereas there was strong WWOX protein expression (d) in the same tissue section (scale bar, $50 \mu \mathrm{m}$ ). (B) Linear regression analysis showed a significant positive correlation between ACK1 and p-ACK1 protein expression. (C) Linear regression analysis showed a significant negative correlation between ACK1 and WWOX protein expression.

explore whether ACK1 amplification is associated with ACK1 activation, we detected the phosphorylated ACK1 expression (phosphorylated at Tyr284, p-ACK1) using immunohistochemical staining, which was previously shown to be associated with ACK1 activation in many studies. As we expected, there was indeed an evident positive correlation between the expression level of ACK1 and p-ACK1 ( $r=0.786$, $\mathrm{P}<0.01$, Spearman's correlation test) (Fig. 4A and B). Our results indicate that $\mathrm{ACK} 1$ amplification is related to the increase in activated p-ACK1.

Since WWOX is downregulated in HCC and is an interacting protein of ACK1, we examined the correlation between
ACK1 and WWOX in 150 pairs of HCC and matched tumoradjacent liver tissues using immunohistochemical staining. IHC scores were used for semiquantitative analysis of ACK1 and WWOX expression. In this study, there was indeed an evident negative correlation between the expression level of ACK 1 and WWOX $(r=-0.454, \mathrm{P}<0.01$, Spearman's correlation test) (Fig. 4A and C).

Knockdown of ACK1 promotes $\mathrm{MHCC}-97 \mathrm{H}$ cell apoptosis and represses invasion, migration and proliferation. To further identify the effect of ACK1 on cell apoptosis, invasion, migration and proliferation of $\mathrm{HCC}$, we downregulated the 
A

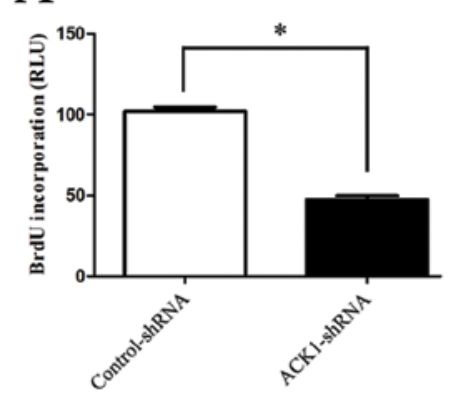

$\mathrm{D}$

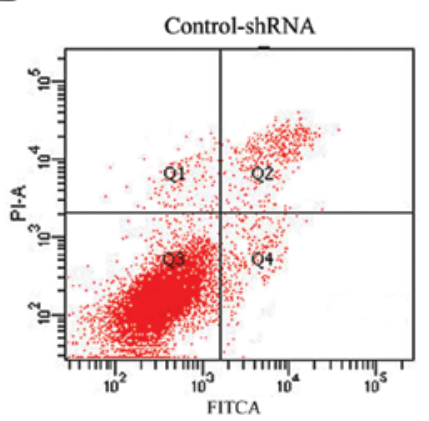

B

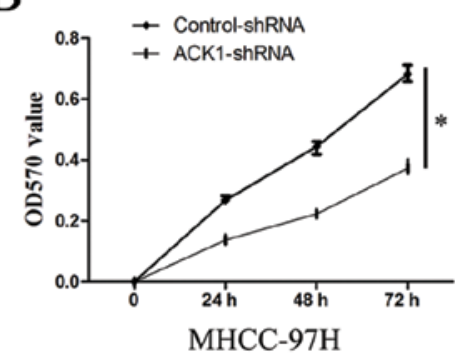

MHCC-97H

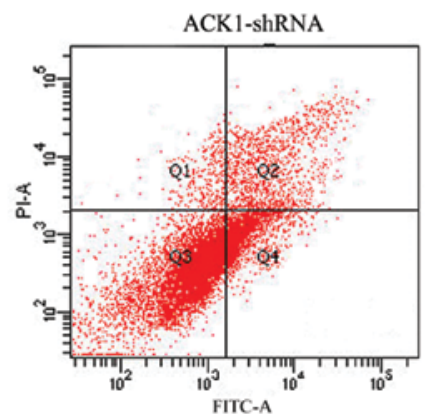

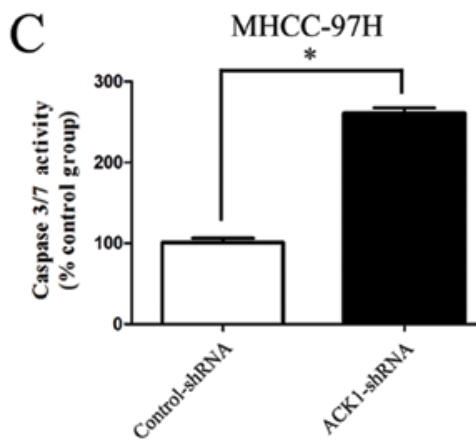

MHCC-97H

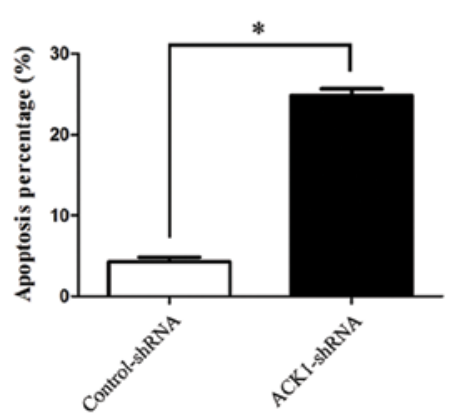

Figure 5. ACK1 regulates apoptosis and proliferation in MHCC-97H cells. (A) Cell proliferation as measured by BrdU incorporation was inhibited by ACK1shRNA in MHCC-97H cells ( $\left.\mathrm{n}=3,{ }^{*} \mathrm{P}<0.01\right)$. (B) As assessed by MTT assay, ACK1 knockdown was found to reduce the viability of MHCC-97H cells ( $\mathrm{n}=3$, $\left.{ }^{*} \mathrm{P}<0.01\right)$. (C) The activity of the pro-apoptotic caspases 3 and 7 was upregulated after ACK1 knockdown in MHCC-97H cells ( $\mathrm{n}=3$, $\left.{ }^{*} \mathrm{P}<0.01\right)$. (D) Quantification of the apoptotic cell population by flow cytometry. ACK1 knockdown MHCC-97H cells were composed of a larger subset of apoptotic cells compared with the control group $(n=3, " P<0.01)$. Values are depicted as mean \pm SEM.
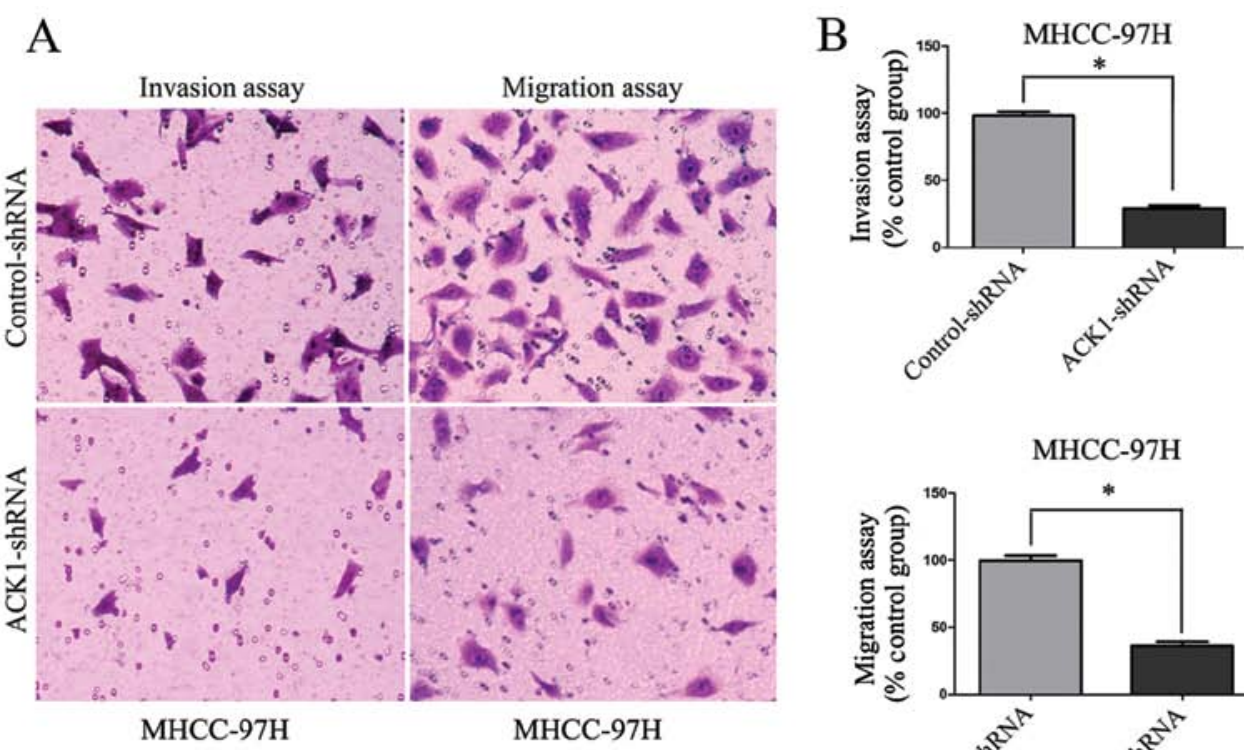

C
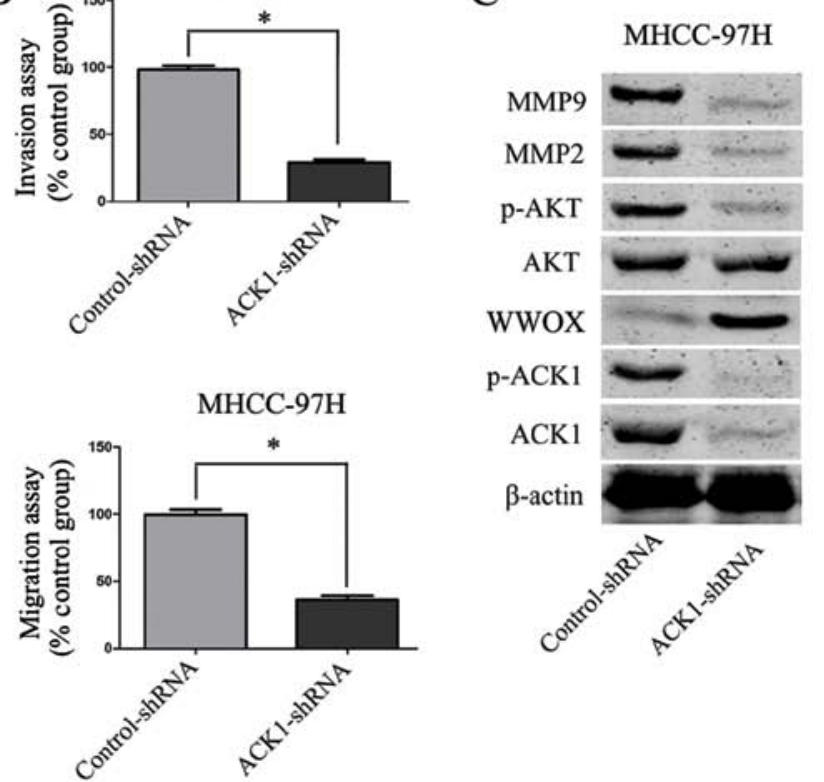

Figure 6. The effect of ACK1 knockdown on MHCC-97H cells. (A) Representative images show the migration and invasion ability of MHCC-97H cells transfected with ACK1-shRNA or Control-shRNA (x200). (B) Data are presented as mean relative numbers of invaded or migrated cells from 5 fields ("P<0.01). (C) MHCC-97H cells transfected with ACK1-shRNA or Control-shRNA, respectively, were subjected to western blotting for ACK1, p-ACK1, WWOX, AKT, p-AKT, MMP2 and MMP9 $(\mathrm{P}<0.01)$.

expression of ACK1 by ACK1-shRNA vectors in MHCC-97H cells. As compared with the control group, silencing ACK1 was found to inhibit MHCC-97H cell proliferation and viability by $\mathrm{BrdU}$ and MTT assays $(\mathrm{P}<0.01$, respectively,
Fig. 5A and B). On the other hand, our study found that the activity of caspase 3/7 in MHCC-97H cells was significantly increased after knockdown of ACK1 $(\mathrm{P}<0.01$, respectively, Fig. 5C). Flow cytometry assay revealed that the percentage of 
Table II. Univariate prognostic analysis of overall survival and disease-free survival in HCC patients.

\begin{tabular}{|c|c|c|c|c|c|c|c|}
\hline \multirow[b]{2}{*}{ Variable } & \multirow[b]{2}{*}{$\mathrm{n}$} & \multicolumn{3}{|c|}{ Overall survival rate (\%) } & \multicolumn{3}{|c|}{ Disease-free survival rate (\%) } \\
\hline & & 3-year & 5 -year & P-value & 3-year & 5 -year & P-value \\
\hline \multicolumn{8}{|l|}{ Gender } \\
\hline Female & 32 & 84.2 & 67.7 & 0.165 & 59.4 & 46.9 & 0.165 \\
\hline Male & 118 & 64.2 & 48.9 & & 51.7 & 35.3 & \\
\hline \multicolumn{8}{|l|}{ Age (year) } \\
\hline$\leq 45$ & 42 & 80.8 & 56.3 & 0.577 & 64.3 & 40.8 & 0.742 \\
\hline$>45$ & 108 & 63.7 & 51.7 & & 49.1 & 36.8 & \\
\hline \multicolumn{8}{|c|}{ HBsAg status ${ }^{\mathrm{a}}$} \\
\hline Negative & 32 & 81.3 & 78.0 & $0.026^{\mathrm{c}}$ & 75.0 & 56.3 & $0.012^{\mathrm{c}}$ \\
\hline Positive & 118 & 64.9 & 45.8 & & 47.5 & 32.8 & \\
\hline \multicolumn{8}{|l|}{ Cirrhosis } \\
\hline No & 48 & 68.7 & 64.2 & 0.143 & 62.5 & 45.3 & 0.078 \\
\hline Yes & 102 & 68.3 & 47.7 & & 49.0 & 34.3 & \\
\hline \multicolumn{8}{|l|}{$\operatorname{AFP}(\mu \mathrm{g} / \mathrm{l})^{\mathrm{b}}$} \\
\hline$\leq 400$ & 67 & 65.3 & 57.3 & 0.289 & 55.2 & 44.5 & 0.350 \\
\hline$>400$ & 83 & 71.0 & 49.2 & & 51.8 & 32.5 & \\
\hline \multicolumn{8}{|l|}{ Tumor size } \\
\hline$\leq 5 \mathrm{~cm}$ & 55 & 68.5 & 54.8 & 0.951 & 49.1 & 37.8 & 0.568 \\
\hline$>5 \mathrm{~cm}$ & 95 & 68.4 & 51.7 & & 55.8 & 37.9 & \\
\hline \multicolumn{8}{|c|}{ Tumor number } \\
\hline Single & 118 & 73.4 & 58.8 & $<0.001^{\mathrm{c}}$ & 59.3 & 44.7 & $<0.001^{\mathrm{c}}$ \\
\hline Multiple & 32 & 50.0 & 31.3 & & 31.3 & 12.5 & \\
\hline \multicolumn{8}{|c|}{ Tumor capsule } \\
\hline Complete & 35 & 73.7 & 60.8 & 0.310 & 57.1 & 40.0 & 0.904 \\
\hline Incomplete & 115 & 66.9 & 50.5 & & 52.2 & 37.1 & \\
\hline \multicolumn{8}{|c|}{ Vascular invasion } \\
\hline No & 128 & 68.4 & 55.1 & $0.036^{\mathrm{c}}$ & 55.5 & 41.2 & $0.018^{\mathrm{c}}$ \\
\hline Yes & 22 & 68.2 & 39.2 & & 40.9 & 18.2 & \\
\hline \multicolumn{8}{|c|}{ Edmondson grade } \\
\hline $\mathrm{I} / \mathrm{II}$ & 91 & 76.8 & 57.6 & $0.020^{c}$ & 67.0 & 48.0 & $<0.001^{\mathrm{c}}$ \\
\hline III/IV & 59 & 55.6 & 45.1 & & 32.2 & 22.0 & \\
\hline \multicolumn{8}{|l|}{ TNM stage } \\
\hline $\mathrm{I} / \mathrm{II}$ & 96 & 71.4 & 61.4 & $0.001^{\mathrm{c}}$ & 60.4 & 46.6 & $0.001^{\mathrm{c}}$ \\
\hline III/IV & 54 & 63.0 & 37.7 & & 40.7 & 22.2 & \\
\hline \multicolumn{8}{|c|}{ ACK1 protein level } \\
\hline Low & 75 & 86.5 & 69.0 & $<0.001^{\mathrm{c}}$ & 76.0 & 55.6 & $<0.001^{\mathrm{c}}$ \\
\hline High & 75 & 50.6 & 36.9 & & 30.7 & 20.0 & \\
\hline
\end{tabular}

${ }^{\mathrm{a}} \mathrm{HbsAg}$, hepatitis B surface antigen; ${ }^{\mathrm{b}} \mathrm{AFP}, \alpha$-fetoprotein; ${ }^{\mathrm{c}} \mathrm{P}<0.05$.

apoptosis in HCC cells in ACK1-shRNA group was increased $(\mathrm{P}<0.01$, respectively, Fig. 5D). Our study also found that knockdown of ACK1 expression significantly reduced the number of migrated and invaded MHCC-97H cells $(\mathrm{P}<0.01$, respectively, Fig. $6 \mathrm{~A}$ and $\mathrm{B})$.
Knockdown of ACK1 is associated with upregulation of WWOX and inactivation of AKT signaling in MHCC-97H cells. To identify the underlying mechanism by which ACK1 regulates tumor cell apoptosis, invasion, migration and proliferation in HCC, we investigated the effect of ACK1 on 
Table III. Multivariate analysis of factors contributing to overall survival and disease-free survival in HCC patients.

\begin{tabular}{|c|c|c|c|c|}
\hline \multirow[b]{2}{*}{ Variable } & \multicolumn{2}{|c|}{ Overall survival rate } & \multicolumn{2}{|c|}{ Disease-free survival rate } \\
\hline & $\mathrm{HR}(95 \% \mathrm{CI})^{\mathrm{b}}$ & P-value & $\mathrm{HR}(95 \% \mathrm{CI})^{\mathrm{b}}$ & P-value \\
\hline HBsAg status $^{\mathrm{a}}$ & $2.512(1.301-4.849)$ & $0.006^{\mathrm{c}}$ & $2.410(1.354-4.290)$ & $0.003^{\mathrm{c}}$ \\
\hline Tumor number & $2.596(1.264-5.333)$ & $0.009^{c}$ & $2.422(1.268-4.628)$ & $0.007^{\mathrm{c}}$ \\
\hline Vascular invasion & $0.654(0.333-1.286)$ & 0.219 & $0.739(0.395-1.384)$ & 0.345 \\
\hline TNM stage & $1.142(0.560-2.329)$ & 0.715 & $1.039(0.564-1.917)$ & 0.902 \\
\hline Edmondson grade & $1.029(0.630-1.680)$ & 0.910 & $1.505(0.983-2.305)$ & 0.060 \\
\hline ACK1 level & $2.523(1.496-4.255)$ & $0.001^{\mathrm{c}}$ & $2.318(1.479-3.634)$ & $<0.001^{\mathrm{c}}$ \\
\hline
\end{tabular}

${ }^{\mathrm{a}} \mathrm{HbsAg}$, hepatitis B surface antigen; ${ }^{\mathrm{b}} \mathrm{HR}$, hazard ratio; CI, confidence interval; ${ }^{\mathrm{c}} \mathrm{P}<0.05$.

p-ACK1, WWOX and AKT signaling. Knockdown of ACK1 evidently increased the level of WWOX and attenuated the phosphorylation of ACK1 and AKT in MHCC-97H cells $(\mathrm{P}<0.01$, Fig. 6C). Furthermore, to better understand the downstream molecules involved in ACK1 induced-AKT signaling activation, we identified whether ACK1 affected MMP2 and MMP9 expression in HCC cells. As expected, we found that knockdown of ACK1 expression markedly decreased the MMP2 and MMP9 expression in MHCC-97H cells $(\mathrm{P}<0.01$, Fig. 6C).

\section{Discussion}

Due to the high frequency of tumor recurrence, the prognosis of patients with HCC remains very poor (2). Many biomarkers have been associated to the progression of $\mathrm{HCC}$, but most of them turned out not to be of clinical utility (6). Thus, it is urgent to explore effective biomarkers to improve the treatment efficacy of HCC. ACK1, an intracellular tyrosine kinase, is considered to be implicated in regulating multiple aspects of tumor progression $(13,16)$. Several studies have indicated that ACK1 overexpression is commonly observed in human cancers including lung and prostate cancer $(27,33)$. Furthermore, it has been demonstrated that ACK1 can promote tumor growth via regulating pro-survival molecules such as AKT and AR in various cancers $(26,31,33)$.

The role of ACK1 have been investigated in multiple tumor types $(31,33)$. However, the ACK1 expression and its related molecule mechanisms in HCC remained unknown. In this study, we initially examined the expression of ACK1 in an immortalized nontumorigenic hepatocyte cell line L02 and five HCC cell lines, such as SMMC-7721, Huh-7, HepG2, BEL-7402 and MHCC-97H. We found that ACK1 expression in immortalized nontumorigenic hepatocyte cell line L02 was lower than that in HCC cell lines. We further investigated ACK1 expression in $150 \mathrm{HCC}$ patients using qRT-PCR, immunohistochemical staining and western blotting, and our results found that the expression level of ACK1 was evidently higher in HCC compared with matched normal tumor adjacent liver tissues. Moreover, ACK1 expression level was significantly correlated with several clinicopathological parameters including Edmondson-Steiner grade, tumor number, vascular invasion and TNM stage. Notably, our results also indicates that high-expression level of ACK1 was evidently correlated with shorter overall survival and disease-free survival, which is an assumed biomarker for HCC negative prognosis. Additionally, multivariate Cox repression analysis further confirmed that ACK1 is an independent factor for HCC patients. Our results revealed that the expression level of ACK1 is a critical factor for prognosis determination and ACK1 may promote tumor progression in HCC.

In this study, we detected the phosphorylated ACK1 expression (p-ACK1), which was previously shown to be associated with ACK1 activation. As expected, our results indicate that ACK1 amplification is related to the increase in activated p-ACK1. Our data are consistent with previous studies on gastric cancer and breast cancer, suggesting the ACK1 gene amplification can cause ACK1 phosphorylation and auto-activation $(15,16)$. It has been demonstrated that ACK1 is responsible for the regulation of several important molecule proteins, which are involved in tumor cell apoptosis, invasion, migration and proliferation, such as WWOX, AKT and AR $(21,23)$. In our study, ACK1 expression was inversely associated with WWOX expression in HCC samples. The in vitro experiments presented in this study also indicate that ACK1 is an important regulatory factor in HCC cell apoptosis, invasion, migration and proliferation. Our in vitro studies also revealed that knockdown of ACK1 by shRNA in MHCC-97H, a cell line with high expression level of the target gene, consistently showed degradation of the p-AKT, MMP2 and MMP9 expression. Moreover, our study confirmed that ACK1 mediates the degradation of WWOX and activates AKT signaling in HCC.

As a recently discovered tumor suppressor, the expression level of WWOX have been shown to be downregulated in various tumor types including $\operatorname{HCC}(19,20)$. WWOX protein plays an inhibitory role in Wnt/ $\beta$-catenin signaling pathway, which is an important signaling for tumor progression (19). High frequency of loss of WWOX is observed in breast, prostate and ovarian cancer (20-22). Additionally, it has been reported that WWOX was identified to be an ACK1 interacting protein and Ack1 was shown to be prerequisite for WWOX protein degradation (23). In this study, we found that ACK1 downregulated WWOX expression, which is consistent with a previous study (23). 
Previously, activated AKT signaling has been considered to facilitate tumor cell proliferation, invasion and migration (24). Multiple studies found that AKT signaling pathway is aberrantly hyperactivated in HCC, which results in the promotion of tumor progression $(6,24)$. Furthermore, it has been reported that AKT signaling plays a key role in facilitating MMP2 and MMP9 expression (6). As two critical members of the MMPs family, MMP2 and MMP9 are important factors in the invasion and migration of HCC (34). In this study, we found that knockdown of ACK1 expression resulted in inactivation of AKT signaling and downregulation the expression of MMP2 and MMP9 in HCC cells. Our data indicate that ACK1 promotes cell invasion and migration through AKT signaling activation and MMP2/9 upregulation and ACK1 may be an important alternate AKT activator in HCC.

In summary, we found that ACK1 expression is upregulated in HCC tissues as compared with noncancerous liver tissues and that overexpression level of ACK1 is correlated with clinicopathological features of poor prognosis in HCC. Additionally, we identified that ACK1 expression is an independent factor for predicting the overall survival and disease-free survival of HCC patients. ACK1 was positively associated with p-ACK1 and was negatively associated with WWOX expression. In vitro studies showed that knockdown of ACK1 promoted HCC cells apoptosis and repressed HCC cell invasion, migration and proliferation. Furthermore, knockdown of ACK1 resulted in upregulation of WWOX and inactivation of AKT signaling. In this study, we also found that knockdown of ACK1 resulted in the downregulation of MMP2 and MMP9 in HCC. Notably, our results indicate that ACK1 is a candidate oncogene in HCC because it plays an important role in hepatocarcinogenesis. This investigation revealed a potential target of ACK1 and supplied us with a new insight into the degradation of WWOX and activation of AKT signaling in HCC. ACK1 may potentially act as a prognostic biomarker, and may also be a therapeutic target in HCC.

\section{Acknowledgements}

This study was supported by grants from the National Natural Science Foundation of China (30600156), the Natural Science Foundation of Jiangxi Province (2012ZBAB205001), the Science and Technology Projects Foundation of Jiangxi Province (20112BBG70037) and the Projects Foundation of Jiangxi Province Education Department (GJJ14681). The authors would like to thank all the patients who participated in this study.

\section{References}

1. Jemal A, Bray F, Center MM, Ferlay J, Ward E and Forman D: Global cancer statistics. CA Cancer J Clin 61: 69-90, 2011.

2. Chen WQ, Zeng HM, Zheng RS, Zhang SW and He J: Cancer incidence and mortality in China, 2007. Chin J Cancer Res 24: $1-8,2012$

3. Nishikawa H, Osaki Y, Endo M, et al: Japanese Red Cross Liver Study Group: Comparison of standard-dose and half-dose sorafenib therapy on clinical outcome in patients with unresectable hepatocellular carcinoma in field practice: A propensity score matching analysis. Int J Oncol 45: 2295-2302, 2014.
4. Venook AP, Papandreou C, Furuse J and de Guevara LL: The incidence and epidemiology of hepatocellular carcinoma: A global and regional perspective. Oncologist 15 (Suppl 4): 5-13, 2010.

5. Alazawi W, Cunningham M, Dearden J and Foster GR: Systematic review: Outcome of compensated cirrhosis due to chronic hepatitis $\mathrm{C}$ infection. Aliment Pharmacol Ther 32: 344-355, 2010.

6. Liu Q, Tu K, Zhang H, Zheng X, Yao Y and Liu Q: TPX2 as a novel prognostic biomarker for hepatocellular carcinoma. Hepatol Res: Sep 29, 2014 (Epub ahead of print). doi: 10.1111/ hepr.12428.

7. Bruix J and Sherman M; American Association for the Study of Liver Diseases: Management of hepatocellular carcinoma: An update. Hepatology 53: 1020-1022, 2011.

8. Han S, Han L, Yao Y, Sun H, Zan X and Liu Q: Activated hepatic stellate cells promote hepatocellular carcinoma cell migration and invasion via the activation of FAK-MMP9 signaling. Oncol Rep 31: 641-648, 2014.

9. Nur-E-Kamal MS, Kamal JM, Qureshi MM and Maruta H: The CDC42-specific inhibitor derived from ACK-1 blocks v-Ha-Rasinduced transformation. Oncogene 18: 7787-7793, 1999.

10. Prieto-Echagüe V, Gucwa A, Craddock BP, Brown DA and Miller WT: Cancer-associated mutations activate the nonreceptor tyrosine kinase Ack1. J Biol Chem 285: 10605-10615, 2010.

11. Chan W, Tian R, Lee YF, Sit ST, Lim L and Manser E: Downregulation of active ACK1 is mediated by association with the E3 ubiquitin ligase Nedd4-2. J Biol Chem 284: 8185-8194, 2009.

12. Grøvdal LM, Johannessen LE, Rødland MS, Madshus IH and Stang E: Dysregulation of Ack1 inhibits down-regulation of the EGF receptor. Exp Cell Res 314: 1292-1300, 2008.

13. Kelley LC and Weed SA: Cortactin is a substrate of activated Cdc42-associated kinase 1 (ACK1) during ligand-induced epidermal growth factor receptor downregulation. PLoS One 7: e44363, 2012.

14. Gajiwala KS, Maegley K, Ferre R, He YA and Yu X: Ack1: Activation and regulation by allostery. PLoS One 8: e53994, 2013.

15. Shinmura K, Kiyose S, Nagura K, Igarashi H, Inoue Y, Nakamura S, Maeda M, Baba M, Konno H and Sugimura H: TNK2 gene amplification is a novel predictor of a poor prognosis in patients with gastric cancer. J Surg Oncol 109: 189-197, 2014.

16. Howlin J, Rosenkvist $\mathbf{J}$ and Andersson T: TNK2 preserves epidermal growth factor receptor expression on the cell surface and enhances migration and invasion of human breast cancer cells. Breast Cancer Res 10: R36, 2008.

17. Mahajan K, Coppola D, Challa S, et al: Ack1 mediated AKT/ PKB tyrosine 176 phosphorylation regulates its activation. PLoS One 5: e9646, 2010.

18. Mahajan K and Mahajan NP: ACK1 tyrosine kinase: Targeted inhibition to block cancer cell proliferation. Cancer Lett 338: 185-192, 2013

19. Li YP, Wu CC, Chen WT, Huang YC and Chai CY: The expression and significance of WWOX and $\beta$-catenin in hepatocellular carcinoma. APMIS 121: 120-126, 2013.

20. Salah Z, Aqeilan R and Huebner K: WWOX gene and gene product: Tumor suppression through specific protein interactions. Future Oncol 6: 249-259, 2010.

21. Yang $\mathbf{J}$ and Zhang W: WWOX tumor suppressor gene. Histol Histopathol 23: 877-882, 2008.

22. Lewandowska U, Zelazowski M, Seta K, Byczewska M, Pluciennik E and Bednarek AK: WWOX, the tumour suppressor gene affected in multiple cancers. J Physiol Pharmacol 60 (Suppl 1): 47-56, 2009.

23. Mahajan K and Mahajan NP: Shepherding AKT and androgen receptor by Ack1 tyrosine kinase. J Cell Physiol 224: 327-333, 2010.

24. Zheng X, Gai X, Ding F, Lu Z, Tu K, Yao Y and Liu Q: Histone acetyltransferase PCAF up-regulated cell apoptosis in hepatocellular carcinoma via acetylating histone $\mathrm{H} 4$ and inactivating AKT signaling. Mol Cancer 12: 96, 2013.

25. Liu Q, Yang P, Tu K, Zhang H, Zheng X, Yao Y and Liu Q: TPX2 knockdown suppressed hepatocellular carcinoma cell invasion via inactivating AKT signaling and inhibiting MMP2 and MMP9 expression. Chin J Cancer Res 26: 410-417, 2014.

26. Mahajan K and Mahajan NP: PI3K-independent AKT activation in cancers: a treasure trove for novel therapeutics. J Cell Physiol 227: 3178-3184, 2012. 
27. Tan DS, Haaland B, Gan JM, et al: Bosutinib inhibits migration and invasion via ACK1 in KRAS mutant non-small cell lung cancer. Mol Cancer 13: 13, 2014.

28. van der Horst EH, Degenhardt YY, Strelow A, et al: Metastatic properties and genomic amplification of the tyrosine kinase gene ACK1. Proc Natl Acad Sci USA 102: 15901-15906, 2005.

29. Mahajan K, Coppola D, Rawal B, Chen YA, Lawrence HR, Engelman RW, Lawrence NJ and Mahajan NP: Ack1-mediated androgen receptor phosphorylation modulates radiation resistance in castration-resistant prostate cancer. J Biol Chem 287: 22112-22122, 2012.

30. Chua BT, Lim SJ, Tham SC, Poh WJ and Ullrich A: Somatic mutation in the ACK1 ubiquitin association domain enhances oncogenic signaling through EGFR regulation in renal cancer derived cells. Mol Oncol 4: 323-334, 2010.
31. Mahajan K, Coppola D, Chen YA, Zhu W, Lawrence HR, Lawrence NJ and Mahajan NP: Ack1 tyrosine kinase activation correlates with pancreatic cancer progression. Am J Pathol 180: 1386-1393, 2012.

32. Cheng CH, Lee CF, Wu TH, Chan KM, Chou HS, Wu TJ, Yu MC, Chen TC, Lee WC and Chen MF: Evaluation of the new AJCC staging system for resectable hepatocellular carcinoma. World J Surg Oncol 9: 114, 2011.

33. Mahajan K, Challa S, Coppola D, Lawrence H, Luo Y, Gevariya $\mathrm{H}$, Zhu W, Chen YA, Lawrence NJ and Mahajan NP: Effect of Ack1 tyrosine kinase inhibitor on ligand-independent androgen receptor activity. Prostate 70: 1274-1285, 2010.

34. Kessenbrock K, Plaks V and Werb Z: Matrix metalloproteinases: Regulators of the tumor microenvironment. Cell 141: 52-67, 2010. 